\title{
Vibsane-type Diterpenes from Taiwanese Viburnum odoratissimum
}

\author{
Miwa Kubo, ${ }^{a}$ Ih-Sheng CHen, ${ }^{b}$ and Yoshiyasu FunuyamA ${ }^{*, a}$ \\ Institute of Pharmacognosy, Faculty of Pharmaceutical Sciences, Tokushima Bunri University, ${ }^{a}$ Yamashiro-cho, Tokushima \\ 770-8514, Japan and School of Pharmacy, Kaohsiung Medical College, ${ }^{b}$ Kaohsing, Taiwan. \\ Received August 21, 2000; accepted November 2, 2000
}

Two new eleven-membered and three new seven-membered vibsane-type diterpenes, named vibsanin $L$ (1) and 14-hydroxyvibsanin $F(2)$, and vibsanin $I(3), 14 R^{*}, 15$-epoxyvibsanin $C$ (4) and $14 S^{*}, 15$-epoxyvibsanin $C$ (5) respectively, have been isolated from Viburnum odoratissimum collected in Taiwan. Their structures have been elucidated by spectroscopic analyses and comparison of Nuclear magnetic resonance method (NMR) data with those of the previously reported vibsane-type diterpenes. High performance liquid chromatography (HPLC) analyses indicated that the methanol extract of $V$. odoratissimum collected in Taiwan contained no neovibsanines, characteristic chemicals occurring in $\mathbf{V}$. awabuki.

Key words Vibsane-type diterpenes; Viburnum odoratissimum; Taiwan; Caprifoliaceae

A number of Viburnum species are known in the world, and among them fifteen species grow in Japan. ${ }^{1)}$ Some Viburnum species have elaborated iridoid glycosides, triterpenes, labdane-type diterpenes, and some aromatic compounds. ${ }^{2-10)}$ The occurrence of vibsane-type diterpenes, however, are limited to $V$. awabuki and has not been so far isolated from other Viburnum species. ${ }^{11-17)}$ These diterpenes can be divided into three subtypes such as 11-membered ring, 7-membered ring, and rearranged types. We have examined chemical components in the leaves of Viburnum odoratissimum collected in Taiwan to find out some of vibsane-type diterpenes. In a preceding paper, ${ }^{18)}$ we already reported the isolation of sevenmembered vibsane-type diterpenes, aldovibsanins $6-8$, which featured the absence of a $\beta, \beta$-dimethylacrylate group at the C-8 position, from the methanol extract of $V$. odoratissimum. Our further chemical studies on this plant have resulted in the isolation of two new eleven-membered and three new seven-membered vibsane-type diterpenes, named vibsanin L (1) and 14-hydroxyvibsanin F (2), and vibsanin I (3), $14 R^{*}, 15$-epoxyvibsanin $\mathrm{C}(4)$ and $14 S^{*}, 15$-epoxyvibsanin $\mathrm{C}$ (5), respectively. Herein, we report the structures of these new compounds and the chemical difference between Viburnum species on the basis of HPLC analyses.

Compound 1 has the molecular formula, $\mathrm{C}_{25} \mathrm{H}_{36} \mathrm{O}_{6}$, established by high-resolution (HR) fast atom bombardment mass spectrometry (FABMS). Its infrared spectroscopy (IR) spectrum showed the presence of a hydroxy group $\left(3356 \mathrm{~cm}^{-1}\right)$ and two carbonyl groups $\left(1700,1719 \mathrm{~cm}^{-1}\right)$. The ${ }^{1} \mathrm{H}-\mathrm{NMR}$ data of 1 showed the signals due to six tertiary methyl groups $\left(\delta_{\mathrm{H}} 1.05,1.46,1.57,1.66,1.91,2.15\right)$, five olefinic protons $\left[\delta_{\mathrm{H}} 5.05(\mathrm{ddq}, J=7.1,7.1,0.8 \mathrm{~Hz}), 5.34(\mathrm{dd}, J=15.9,9.6\right.$ $\mathrm{Hz}), 5.66$ (d, $J=15.9 \mathrm{~Hz}), 5.71$ (qq, $J=1.4,1.4 \mathrm{~Hz}), 5.84$ (dd, $J=11.0,6.6 \mathrm{~Hz})]$, three oxymethines $\left[\delta_{\mathrm{H}} 3.33(\mathrm{~d}, J=8.7 \mathrm{~Hz})\right.$, $4.21(\mathrm{~d}, J=8.7 \mathrm{~Hz}), 5.11(\mathrm{~d}, J=9.6 \mathrm{~Hz})]$, and an oxymethylene $\left[\delta_{\mathrm{H}} 4.21(\mathrm{~d}, J=13.2 \mathrm{~Hz}), 4.67(\mathrm{~d}, J=13.2 \mathrm{~Hz})\right]$. Extensive analysis of ${ }^{1} \mathrm{H}-{ }^{1} \mathrm{H}$ correlated spectroscopy (COSY) and heteronuclear multiple quantum coherence (HMQC) gave the four partial structures $\mathbf{A}-\mathbf{F}$ as depicted by bold lines in Fig. 1 and three quaternary carbons at $\delta_{\mathrm{C}} 48.0,62.3$ and 201.6. The double bond involved in the $\mathbf{B}$ unit should take $E$-geometry due to a large vicinal coupling constant $(J=15.9 \mathrm{~Hz})$. In order to determine the connectivities between these six partial structures and three quaternary carbons, heteronuclear multiple bond correlation (HMBC) experiments were carried out. The HMBC correlation of $\mathrm{H}-8$ to $\mathrm{C}-1^{\prime}$ linked the $\beta, \beta$ acrylate ester group $\mathbf{A}$ to $\mathbf{C}-8$ in the partial structure $\mathbf{D}$. The correlation of $\mathrm{H}-18$ to $\mathrm{C}-3$ supported the linkage between $\mathrm{C}$ 18 and C-3, thereby assembling the units $\mathbf{D}$ and E. The C-4 carbonyl carbon resonated at $\delta_{\mathrm{C}} 201.6$ showed cross peaks to the H-2, H-18 and H-5 signals, thus making two bonds not only between $\mathrm{C}-4$ and $\mathrm{C}-3$ in the unit $\mathbf{E}$, but also between $\mathrm{C}$ 4 and $\mathrm{C}-5$ in the unit $\mathbf{C}$. The quaternary carbon $\mathrm{C}-11$ showed cross peaks to $\mathrm{H}-1, \mathrm{H}_{3}-20$ and $\mathrm{H}-12$, respectively, and also $\mathrm{H}-$ 10 correlated to $\mathrm{C}-11$, resulting in the formation of the quaternary carbon $\mathrm{C}-11$ with $\mathrm{C}-1, \mathrm{C}-12, \mathrm{C}-10$ and C-20. Additionally, the HMBC correlations of $\mathrm{H}-6, \mathrm{H}-8$ and $\mathrm{H}_{3}-19$ to $\mathrm{C}-$ $7\left(\delta_{\mathrm{C}} 62.3\right)$ showed that C-7 connected to both C-6 of the unit $\mathrm{C}$ and $\mathrm{C}-8$ of the unit $\mathbf{B}$. These HMBC correlations led to the construction of an eleven membered ring. Taking into consideration eight degrees of unsaturation and high-field ${ }^{13} \mathrm{C}-\mathrm{NMR}$ chemical shifts $\left(\delta_{\mathrm{C}} 63.4\right.$ and 62.3 ) of C-6 and C-7 in comparison with those of normal oxygen-bearing carbons, an epoxide ring must be formed at the C-6 and C-7 positions. Thus, the above spectral data gave the planar structure $\mathbf{1}$ as shown in Fig. 1.

The relative stereochemistry of $\mathbf{1}$ was elucidated by nuclear Overhauser exchange spectroscopy (NOESY) as shown in Fig. 2. Namely, the H-9 and H-6 signals showed cross peaks to the $\mathrm{H}_{3}-19$ and $\mathrm{H}_{3}-20$, and $\mathrm{H}_{3}-19$ resonances, respectively, indicating that both the C-19 and C-20 methyl groups took the same $\beta$-configuration. The observation of cross peaks from $\mathrm{H}-5$ to $\mathrm{H}-6, \mathrm{H}-9$ and $\mathrm{H}-18$ as well as from $\mathrm{H}-10$ to $\mathrm{H}-12$ and $\mathrm{H}-8$, disclosed that the $\beta, \beta$-dimethylacryl group on the C-8 position and the C-19 methyl group aligned with the same spatial arrangement, and both the hydroxy group on the $\mathrm{C}-5$ position and the epoxide ring took the same $\alpha$ orientation. Additionally, the $Z$ geometry of $\Delta^{2,3}$ double bond is evident of NOE between H-18 and H-2. On the basis of the above NOE analyses, the structure of vibsanin L was elucidated as $\mathbf{1}$.

Compound 2 had the molecular formula $\mathrm{C}_{20} \mathrm{H}_{32} \mathrm{O}_{3}$ as established by HR-FABMS. The spectral data of $\mathbf{2}$ were very similar to those of an 11-membered vibsane-type diterpene, vibsanin $\mathrm{F}$ (2a), ${ }^{11)}$ except for the $\mathrm{C}-12-\mathrm{C}-17$ side chain. In fact, the ${ }^{1} \mathrm{H}-\mathrm{NMR}$ spectrum of $\mathbf{2}$ showed the signals were due to an exomethylene group [ $\delta_{\mathrm{H}} 4.84(\mathrm{dq}, J=3.2,1.6 \mathrm{~Hz}), 4.93$ 


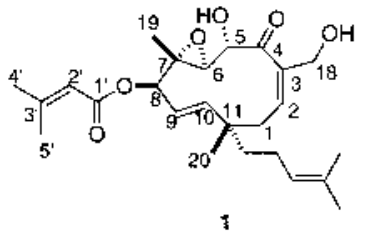

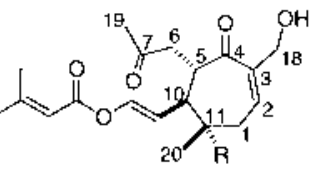

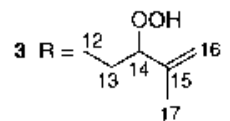

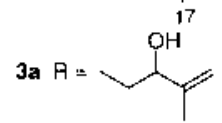<smiles>CCCCCCCCCC=C(C)C</smiles><smiles>CC(C)=CCC1(C)CC=C(CO)C2(O)CC(C)=C(C)CC12C</smiles>

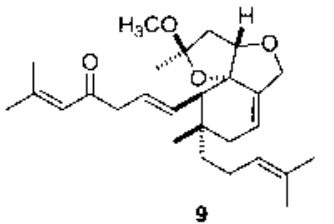

Chart 1

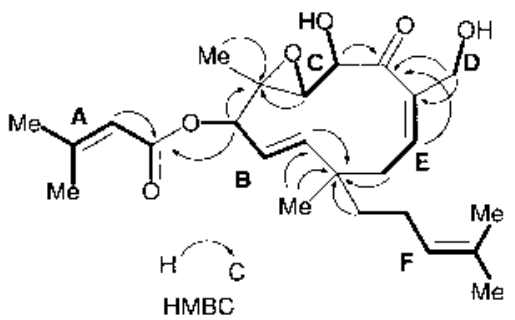

Fig. 1. HMBC Correlations of $\mathbf{1}$

(dq, $J=1.6,1.1 \mathrm{~Hz})]$ and oxymethine proton $\left[\delta_{\mathrm{H}} 4.02\right.$ (brdd, $J=12.4,4.1 \mathrm{~Hz}$ )] instead of the isopropenyl group existing in 2a. In comparison of ${ }^{13} \mathrm{C}-\mathrm{NMR}$ data between 2 and $\mathbf{2 a}$ (Table 1) the $\mathrm{C}-12-\mathrm{C}-17$ side chain of $\mathbf{2}$ was verified to consist of the $\Delta^{15,16}$ double bond and the $\mathrm{C}-14$ methine having a hydroxy group. Additionally, the relative stereochemistry of $\mathbf{2}$ was assigned as the same as that of vibsanin F (2a) by the NOESY experiment. The absolute configuration on C-14 has not been determined. Thus, the structure of $\mathbf{2}$ was represented as 14-hydroxyvibsanin F.

The spectral data of $\mathbf{3}$ were found to be very similar to those of a seven membered vibsane-type diterpene, vibsanin $\mathrm{G}$ (3a). ${ }^{13)}$ The molecular formula of $\mathrm{C}_{25} \mathrm{H}_{36} \mathrm{O}_{7}$ for 3 suggested the presence of one more oxygen atom than $3 \mathbf{a}$, implying the presence of a hydroperoxy group on the $\mathrm{C}-14$ position. In fact, the ${ }^{13} \mathrm{C}$-NMR data of $\mathbf{3}$ were very similar to

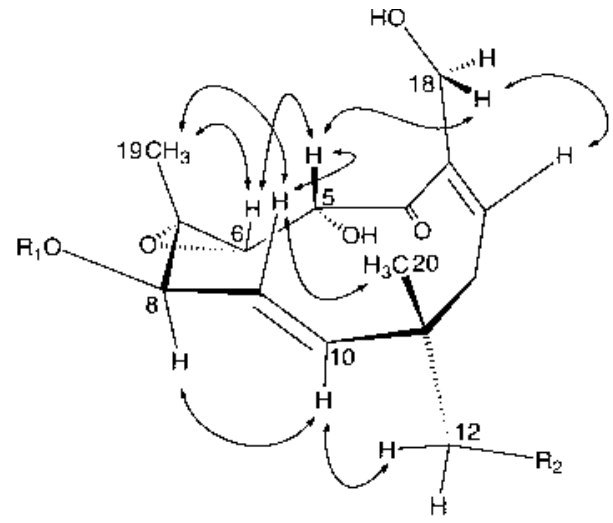

Fig. 2. Conformation of $\mathbf{1}$ Based on NOE Correlations

those of 3a, except the low-field appearance of the C-14 carbon signal appeared at $\delta_{\mathrm{C}} 87.8$ (Table 2). This supported the location of the hydroperoxide group on the $\mathrm{C}-14$ position. The relative stereochemistry of $\mathbf{3}$ was verified to be the same as that of $\mathbf{3 a}$ by the NOESY experiment.

Compounds $\mathbf{4}$ and $\mathbf{5}$ had the same molecular formula $\mathrm{C}_{25} \mathrm{H}_{36} \mathrm{O}_{6}$ established by HR-FABMS. Both of the ${ }^{1} \mathrm{H}-\mathrm{NMR}$ data were not only very similar to each other, but also closely related to those of vibsanin $\mathrm{C}(\mathbf{3 b})^{14)}$ except for the signals due to $\mathrm{H}-12-\mathrm{H}-17$. In fact, the ${ }^{13} \mathrm{C}-\mathrm{NMR}$ data at $\delta_{\mathrm{C}} 63.7$ and 57.1 for 4 and $\delta_{\mathrm{C}} 63.7$ and 57.5 for 5 were assignable to $\mathrm{C}-14$ and $\mathrm{C}-15$, indicating the presence of an epoxide ring on the $\mathrm{C}-14$ and $\mathrm{C}-15$ positions in $\mathbf{4}$ and $\mathbf{5}$. These spectral data suggested that 4 and 5 were 14,15-epoxyvibsanin C. However, the absolute stereochemistry regarding $\mathrm{C}-14$ failed to be clarified. Therefore, we tentatively assigned $\mathbf{4}$ and $\mathbf{5}$ as $14 R^{*}, 15$-epoxyvibsanin $\mathrm{C}$ and $14 S^{*}, 15$-epoxyvibsanin $\mathrm{C}$, respectively.

Viburnum odoratissimum is taxonomically placed as the plant identical to $V$. awabuki. ${ }^{19)}$ Vibsane-type diterpenes have not been so far found in the terrestrial plant, except for $V$. awbuki. In this study, we isolated a number of vibsanetype diterpenes from Taiwanese $V$. odoratissimum. As it was thought to be of interest to chemically compare the methanol extract of $V$. awabuki in Tokushima and $V$. odoratissimum in Taiwan, the analyses of them were performed by HPLC. We found that the two plants differed in the subclass of vibsanetype diterpene as follows; typical 11-membered and 7-membered vibsane-type diterpenes such as vibsanins A, B and $\mathrm{F}^{11)}$ are present in the Viburnum plants in both Tokushima and Taiwan, whereas no neovibsane-type diterpenes such as 9 has been so far found in $V$. odoratissimum. But aldovibsanins, $\mathbf{6}-\mathbf{8}$ which seem to be chemical components characteristic to $V$. odoratissimum, have not been detected from $V$. awabuki.

\section{Experimental}

Optical rotations were measured with a Jasco DIP-1000 digital polarimeter. UV spectra were recorded on a Shimadzu UV-300 or Hitachi-U-3000 spectrophotometer. IR spectra were recorded on a Jasco FT-IR 5300 or FTIR 410 infrared spectrophotometer. 1D- and 2D-NMR spectra were recorded on a Varian Unity 600. MS were recorded on a JEOL AX-500 instrument. Silica gel (Merck, 70-230, 230 - 400 mesh, Wacogel C-300) and octadecylsilica gel (Cosmosil $75 \mathrm{C}_{18}$-OPN) were used for column chromatography. Sephadex LH-20 was used for gel filtration chromatograhy. Precoated Silica gel $60 \mathrm{~F}_{254}$ and RP-8 $\mathrm{F}_{254}$ plates were used for analytical or preparative thinlayer chromatography, and spots were visualized by UV $(254 \mathrm{~nm})$ light and 
Table 1. ${ }^{13} \mathrm{C}-(150 \mathrm{MHz})$ and ${ }^{1} \mathrm{H}-(600 \mathrm{MHz})$ NMR Data of $\mathbf{1}^{a)}, \mathbf{2}^{b)}$, and $\mathbf{2} \mathbf{a}^{b)}$

\begin{tabular}{|c|c|c|c|c|c|}
\hline \multirow{2}{*}{ No. } & \multicolumn{2}{|r|}{1} & \multicolumn{2}{|r|}{2} & \multirow{2}{*}{$\frac{2 \mathbf{a}}{{ }^{13} \mathrm{C}}$} \\
\hline & ${ }^{13} \mathrm{C}$ & ${ }^{1} \mathrm{H}$ & ${ }^{13} \mathrm{C}$ & ${ }^{1} \mathrm{H}$ & \\
\hline 1 & 40.5 & $\begin{array}{l}2.00(\mathrm{dd}, 12.6,6.6) \\
2.55(\mathrm{dd}, 12.6,1.0)\end{array}$ & 42.1 & $\begin{array}{l}1.93(\mathrm{ddd}, 13.2,11.0,6.6) \\
2.22(\mathrm{ddd}, 13.2,11.8,3.0)\end{array}$ & 42.6 \\
\hline 2 & 132.3 & $5.84(\mathrm{dd}, 11.0,6.6)$ & 112.9 & $5.52(\mathrm{dd}, 11.8,11.0)$ & 119.8 \\
\hline 3 & 145.1 & & 141.7 & & 142.4 \\
\hline 4 & 201.6 & & 23.6 & $\begin{array}{l}2.05(\mathrm{dd}, 12.4,7.8) \\
2.33(\mathrm{dd}, 12.4,11.8)\end{array}$ & 24.1 \\
\hline 5 & 72.7 & $4.21(\mathrm{~d}, 8.7)$ & 26.9 & $\begin{array}{l}1.08(\mathrm{~m}) \\
2.27 \text { (br dd, } 11.0,10.2)\end{array}$ & 27.5 \\
\hline 6 & 63.4 & $3.33(\mathrm{~d}, 8.7)$ & 63.4 & $2.72(\mathrm{~d}, 11.0)$ & 63.1 \\
\hline 7 & 62.3 & & 60.5 & & 59.8 \\
\hline 8 & 76.8 & $5.11(\mathrm{~d}, 9.6)$ & 39.0 & $\begin{array}{l}2.09(\mathrm{dd}, 13.2,2.7) \\
2.54(\mathrm{dd}, 13.2,3.4)\end{array}$ & 39.5 \\
\hline 9 & 123.0 & $5.34(\mathrm{dd}, 15.9,9.6)$ & 121.1 & $5.41(\mathrm{ddd}, 10.7,3.4,2.7)$ & 121.4 \\
\hline 10 & 145.7 & $5.66(d, 15.9)$ & 143.3 & $5.44(\mathrm{~d}, 10.7)$ & 143.5 \\
\hline 11 & 48.0 & & 40.5 & & 39.9 \\
\hline 12 & 38.6 & $\begin{array}{l}1.28(\mathrm{ddd}, 13.2,11.8,5.2) \\
1.38(\mathrm{ddd}, 13.2,13.2,4.7)\end{array}$ & 35.3 & $\begin{array}{l}1.36(\mathrm{ddd}, 9.3,7.1,3.6) \\
1.54(\mathrm{ddd}, 9.3,9.3,3.0)\end{array}$ & 40.1 \\
\hline 13 & 23.1 & $\begin{array}{l}1.78(\mathrm{~m}) \\
1.91(\mathrm{~m})\end{array}$ & 29.8 & $\begin{array}{l}1.32(\mathrm{~m}) \\
1.51(\mathrm{~m})\end{array}$ & 23.7 \\
\hline 14 & 124.2 & 5.05 (br ddq, $7.1,7.1,0.8)$ & 76.5 & $4.02(\mathrm{dd}, 12.4,4.1)$ & 125.5 \\
\hline 15 & 131.7 & & 147.5 & & 130.9 \\
\hline 16 & 17.5 & $1.57(\mathrm{~s})$ & 111.0 & $\begin{array}{l}4.84(\mathrm{dq}, 3.2,1.6) \\
4.93(\mathrm{dq}, 1.6,1.1)\end{array}$ & 25.3 \\
\hline 17 & 25.7 & $1.66(\mathrm{~s})$ & 17.4 & $1.71(\mathrm{dd}, 3.2,1.1)$ & 17.7 \\
\hline 18 & 64.8 & $\begin{array}{l}4.21(\mathrm{~d}, 13.2) \\
4.67(\mathrm{~d}, 13.2)\end{array}$ & 66.2 & $\begin{array}{l}4.02(\mathrm{dd}, 12.6,6.6) \\
4.10(\mathrm{dd}, 12.6,3.0)\end{array}$ & 65.4 \\
\hline 19 & 17.6 & $1.46(\mathrm{~s})$ & 23.3 & $1.41(\mathrm{~s})$ & 23.4 \\
\hline 20 & 23.4 & $1.05(\mathrm{~s})$ & 23.7 & $1.08(\mathrm{~s})$ & 23.8 \\
\hline $1^{\prime}$ & 163.2 & & & & \\
\hline $2^{\prime}$ & 114.9 & $5.71(\mathrm{qq}, 1.4,1.4)$ & & & \\
\hline $3^{\prime}$ & 160.3 & & & & \\
\hline $4^{\prime}$ & 20.3 & $2.15(\mathrm{~d}, 1.4)$ & & & \\
\hline $5^{\prime}$ & 27.0 & $1.91(\mathrm{~d}, 1.4)$ & & & \\
\hline
\end{tabular}

a) $\mathrm{CDCl}_{3}$. b) $\mathrm{C}_{6} \mathrm{D}_{6}$.

Table 2. ${ }^{13} \mathrm{C}-\left(150 \mathrm{MHz}, \mathrm{C}_{6} \mathrm{D}_{6}\right)$ and ${ }^{1} \mathrm{H}-\left(600 \mathrm{MHz}, \mathrm{C}_{6} \mathrm{D}_{6}\right)$ NMR Data of $\mathbf{3}, \mathbf{4}$, and 5

\begin{tabular}{|c|c|c|c|c|c|c|}
\hline \multirow{2}{*}{ No. } & \multicolumn{2}{|r|}{3} & \multicolumn{2}{|r|}{4} & \multicolumn{2}{|r|}{5} \\
\hline & ${ }^{13} \mathrm{C}$ & ${ }^{1} \mathrm{H}$ & ${ }^{13} \mathrm{C}$ & ${ }^{1} \mathrm{H}$ & ${ }^{13} \mathrm{C}$ & ${ }^{1} \mathrm{H}$ \\
\hline 1 & 35.7 & $\begin{array}{l}1.86(\mathrm{dd}, 13.2,4.4) \\
1.63(\mathrm{dd}, 13.2,9.1)\end{array}$ & 35.7 & $\begin{array}{l}1.66(\mathrm{dd}, 16.3,8.8) \\
1.84(\mathrm{dd}, 16.3,4.9)\end{array}$ & 35.6 & $\begin{array}{l}1.74(\mathrm{dd}, 15.9,9.1) \\
1.85(\mathrm{dd}, 15.9,5.2)\end{array}$ \\
\hline 2 & 38.4 & $6.06(\mathrm{dd}, 9.1,4.4)$ & 136.4 & $6.09(\mathrm{dd}, 8.8,4.9)$ & 136.5 & $6.12(\mathrm{dd}, 9.1,5.2)$ \\
\hline 3 & 160.3 & & 143.0 & & 143.2 & \\
\hline 4 & 205.3 & & 205.1 & & 204.9 & \\
\hline 5 & 49.1 & $2.88(\mathrm{ddd}, 8.5,4.1,2.4)$ & 48.5 & $2.84(\mathrm{ddd}, 9.9,7.4,4.4)$ & 48.3 & $2.88(\mathrm{ddd}, 10.2,6.7,4.7)$ \\
\hline 6 & 44.3 & $\begin{array}{l}2.77(\mathrm{dd}, 17.9,2.2) \\
2.38(\mathrm{dd}, 17.9,4.1)\end{array}$ & 44.3 & $\begin{array}{l}2.50(\mathrm{dd}, 17.9,4.4) \\
2.75(\mathrm{dd}, 17.9,7.4)\end{array}$ & 44.2 & $\begin{array}{l}2.50(\mathrm{dd}, 17.9,4.7) \\
2.78(\mathrm{dd}, 17.9,6.7)\end{array}$ \\
\hline 7 & 206.4 & & 206.4 & & 206.3 & \\
\hline 8 & 137.6 & $7.14(\mathrm{~d}, 12.4)$ & 137.3 & $7.17(\mathrm{~d}, 12.4)$ & 137.8 & $7.17(\mathrm{~d}, 12.4)$ \\
\hline 9 & 113.9 & $5.08(\mathrm{dd}, 12.4,11.3)$ & 113.3 & $5.10(\mathrm{dd}, 12.4,11.3)$ & 113.3 & $5.12(\mathrm{dd}, 12.4,11.3)$ \\
\hline 10 & 48.0 & $1.90(\mathrm{dd}, 12.4,11.3)$ & 46.4 & $2.06(\mathrm{dd}, 11.3,9.9)$ & 46.2 & $2.09(\mathrm{dd}, 11.3,10.2)$ \\
\hline 11 & 39.9 & & 39.8 & & 39.7 & \\
\hline 12 & 34.5 & $1.05(\mathrm{ddd}, 12.9,12.4,3.0)$ & 36.4 & $1.20(\mathrm{~m})$ & 36.3 & $1.12(\mathrm{~m})$ \\
\hline 13 & 24.9 & $\begin{array}{l}1.32 \text { (dddd, } 12.9,12.6,5.6,3.6 \text { ) } \\
1.43 \text { (dddd, } 12.6,12.6,5.6,3.0 \text { ) }\end{array}$ & 24.4 & $\begin{array}{l}1.16(\mathrm{~m}) \\
1.28(\mathrm{~m})\end{array}$ & 24.2 & $\begin{array}{l}1.12(\mathrm{~m}) \\
1.28(\mathrm{~m})\end{array}$ \\
\hline 14 & 87.8 & $4.26(\mathrm{dd}, 5.6,5.6)$ & 63.7 & $2.39(\mathrm{dd}, 6.0,5.7)$ & 63.7 & $2.39(\mathrm{dd}, 6.3,5.5)$ \\
\hline 15 & 143.5 & & 57.1 & & 57.5 & \\
\hline 16 & 113.2 & $\begin{array}{l}4.90(\mathrm{br} \mathrm{s}) \\
5.00(\mathrm{brs})\end{array}$ & 24.9 & $1.10(\mathrm{~s})$ & 24.9 & $1.10(\mathrm{~s})$ \\
\hline 17 & 18.3 & 1.63 (brs) & 18.7 & $1.12(\mathrm{~s})$ & 18.7 & $1.08(\mathrm{~s})$ \\
\hline 18 & 64.2 & $\begin{array}{l}4.02(\mathrm{brd}, 12.9) \\
4.37(\mathrm{brd}, 12.9)\end{array}$ & 63.6 & $\begin{array}{l}4.28(\mathrm{~d}, 13.0) \\
4.36(\mathrm{~d}, 13.0)\end{array}$ & 63.5 & $\begin{array}{l}4.30(\mathrm{~d}, 13.1) \\
4.41(\mathrm{~d}, 13.1)\end{array}$ \\
\hline 19 & 29.5 & $1.74(\mathrm{~s})$ & 29.5 & $1.75(\mathrm{~s})$ & 29.5 & $1.77(\mathrm{~s})$ \\
\hline 20 & 29.5 & $0.61(\mathrm{~s})$ & 24.2 & $0.60(\mathrm{~s})$ & 23.7 & $0.60(\mathrm{~s})$ \\
\hline $1^{\prime}$ & 163.2 & & 163.1 & & 163.1 & \\
\hline $2^{\prime}$ & 114.9 & $5.64(\mathrm{qq}, 1.4,1.4)$ & 114.8 & $5.64(\mathrm{qq}, 1.4,1.1)$ & 114.9 & $5.63(\mathrm{qq}, 1.1,1.1)$ \\
\hline $3^{\prime}$ & 160.3 & & 160.3 & & 160.2 & \\
\hline $4^{\prime}$ & 20.3 & $1.36(\mathrm{brd}, 1.4)$ & 20.3 & $2.02(\mathrm{brd}, 1.4)$ & 20.2 & $2.01(\mathrm{~d}, 1.1)$ \\
\hline $5^{\prime}$ & 27.0 & $2.02(\mathrm{brd}, 1.4)$ & 27.0 & $1.36(\mathrm{brd}, 1.1)$ & 27.0 & $1.35(\mathrm{~d}, 1.1)$ \\
\hline
\end{tabular}


$2 \% \mathrm{CeSO}_{4}$ in $\mathrm{H}_{2} \mathrm{SO}_{4}$ after heating.

Plant Material The leaves of Viburnum odoratissimum were collected in Taiwan in March, 1999. A voucher specimen was deposited in our institute.

Extraction and Isolation Air-dried and powdered leaves $(1.5 \mathrm{~kg})$ of $V$. odoratissimum were immersed in $\mathrm{MeOH}$ at room temperature for $30 \mathrm{~d}$. The $\mathrm{MeOH}$ extract was concentrated in vacuo to give a gummy extract $(600 \mathrm{~g})$. The $\mathrm{MeOH}$ extract $(384 \mathrm{~g}$ ) was mixed with silica gel (Merck, 70-230 mesh, $220 \mathrm{~g}$ ) and then the $\mathrm{MeOH}$ was removed under reduced pressure. The obtained solids were pulverized, packed into a glass column, and eluted in order with $\mathrm{CH}_{2} \mathrm{Cl}_{2}(21), \mathrm{CH}_{2} \mathrm{Cl}_{2}:$ EtOAc $=9: 1(21), \mathrm{CH}_{2} \mathrm{Cl}_{2}: \mathrm{EtOAc}=3: 2$ (21), $\mathrm{CH}_{2} \mathrm{Cl}_{2}: \mathrm{EtOAc}=1: 1$ (21), $\mathrm{CH}_{2} \mathrm{Cl}_{2}: \mathrm{EtOAc}=2: 3$ (21), EtOAc (21), EtOAc : $\mathrm{MeOH}=9: 1$ (21), EtOAc $: \mathrm{MeOH}=3: 2$ (21), EtOAc $: \mathrm{MeOH}=1: 1$ (21), EtOAc: $\mathrm{MeOH}=2: 3$ (21), and $\mathrm{MeOH}$ (21) to give fractions 1-7. Fraction $5(2.8 \mathrm{~g})$ was purified by a combination of silica gel column chromatography $\left(\mathrm{C}-300, \mathrm{CHCl}_{3}: \mathrm{EtOAc}=1: 1\right)$ and gel filtration $(\mathrm{LH}-20$, hexane: $\mathrm{CHCl}_{3}=3: 2$ ) and finally by preparative TLC (Benzen:EtOAc= 7:3) to give vibsanin L (1) (3.4 mg). Fraction 6 was subjected to reversedphase chromatography using Cosmosil $75 \mathrm{C}_{18}$-OPN (150 g) and eluted with $\mathrm{MeOH}: \mathrm{H}_{2} \mathrm{O}=2: 1$ to give fractions $8-15$. Fraction 14 was purified by column chromatography over silica gel (Merck, 230 - 400 mesh, benzene: $\mathrm{EtOAc}=1: 1)$ to give 14-hydroxyvibsanin $\mathrm{F}(2)(5 \mathrm{mg})$. Fraction 11 was subjected to medium-pressure chromatography (Lobar RP-18, MeOH: $\mathrm{H}_{2} \mathrm{O}=$ $3: 1$ ) and finally purified by HPLC [Cosmosil $5 \mathrm{C}_{18}$-AR, i.d. $10 \times 250 \mathrm{~mm}$; $\left.\mathrm{MeOH}: \mathrm{H}_{2} \mathrm{O}: \mathrm{CH}_{3} \mathrm{CN}=2: 1: 1 ; 2 \mathrm{ml} / \mathrm{min} ; 254 \mathrm{~nm}\right]$ to give vibsanin I (3) (3.6 mg). Fraction $12(1.5 \mathrm{~g})$ was purified by column chromatography over silica gel $(\mathrm{C}-300$, benzene : $\mathrm{EtOAc}=7: 3)$ and then by HPLC [Cosmosil $5 \mathrm{C}_{18}$-AR, i.d. $10 \times 250 \mathrm{~mm} ; \mathrm{MeOH}: \mathrm{H}_{2} \mathrm{O}: \mathrm{CH}_{3} \mathrm{CN}=34: 33: 33 ; 2 \mathrm{ml} / \mathrm{min}$; $254 \mathrm{~nm}$ ] to give $14 R^{*}, 15$-epoxyvibsanin C (4) (3 mg) and $14 S^{*}, 15$-epoxyvibsanin C (5) (2 mg). The previously known vibsanin B ( $818 \mathrm{mg})$, vinsainin $\mathrm{F}$ (2a) $(18 \mathrm{mg})$ and vibsanin A (15 mg) were obtained from fraction 18 .

Vibsanin L (1) Colorless oil; $[\alpha]_{\mathrm{D}}^{20}-61.6^{\circ}\left(c=0.20, \mathrm{CHCl}_{3}\right)$; IR $v_{\max }^{\mathrm{FT}}$ : $3356(\mathrm{OH}), 1719,1700(\mathrm{C}=\mathrm{O}), 1649(\mathrm{C}=\mathrm{C}) \mathrm{cm}^{-1} ; \mathrm{UV} \lambda_{\max }^{\mathrm{EtOH}} \mathrm{nm}: 218(\varepsilon$ 14000); FAB-MS: $m / z 455[\mathrm{M}+\mathrm{Na}]^{+}, 471[\mathrm{M}+\mathrm{K}]^{+}$; HR-FAB-MS: Calcd 455.2410 for $\mathrm{C}_{25} \mathrm{H}_{36} \mathrm{O}_{6} \mathrm{Na}$. Found 455.2431.; ${ }^{1} \mathrm{H}$ - and ${ }^{13} \mathrm{C}-\mathrm{NMR}$ : Table 1.

14-Hydroxyvibsanin F (2) Colorless oil; $[\alpha]_{\mathrm{D}}^{20}+9.1^{\circ}\left(c=018, \mathrm{CHCl}_{3}\right)$; IR $v_{\mathrm{max}}^{\mathrm{FT}}: 3376(\mathrm{OH}) \mathrm{cm}^{-1}$; FAB-MS: $m / z 343[\mathrm{M}+\mathrm{Na}]^{+}, 359[\mathrm{M}+\mathrm{K}]^{+}$; HRFAB-MS: Calcd 343.2250 for $\mathrm{C}_{20} \mathrm{H}_{32} \mathrm{O}_{3} \mathrm{Na}$. Found 343.2252.; ${ }^{1} \mathrm{H}$ and ${ }^{13} \mathrm{C}-$ NMR: Table 1

Vibsanin I (3) Colorless oil; $[\alpha]_{\mathrm{D}}^{20}+73.6^{\circ}\left(c=0.56, \mathrm{CHCl}_{3}\right)$; IR $v_{\text {max }}^{\mathrm{FT}}$ : $3420(\mathrm{OH}), 1715(\mathrm{C}=\mathrm{O}), 1645(\mathrm{C}=\mathrm{C}) \mathrm{cm}^{-1}$; UV $\lambda_{\max }^{\mathrm{EtOH}} \mathrm{nm}: 252(\varepsilon 4300)$; FAB-MS: $m / z 471[\mathrm{M}+\mathrm{Na}]^{+}, 487[\mathrm{M}+\mathrm{K}]^{+}$; HR-FAB-MS: Calcd 471.2359 for $\mathrm{C}_{25} \mathrm{H}_{36} \mathrm{O}_{7} \mathrm{Na}$. Found 471.2357.; ${ }^{1} \mathrm{H}$ - and ${ }^{13} \mathrm{C}$-NMR: Table 2.

14R $\boldsymbol{R}^{*}, \mathbf{1 5}$-Epoxyvibsanin $\mathrm{C}$ (4) Colorless oil; $[\alpha]_{\mathrm{D}}^{20}+97.0^{\circ}(c=0.12$, $\left.\mathrm{CHCl}_{3}\right)$; IR $v_{\text {max }}^{\mathrm{FT}}: 3445(\mathrm{OH}), 1728(\mathrm{C}=\mathrm{O}), 1649(\mathrm{C}=\mathrm{C}) \mathrm{cm}^{-1}$; UV $\lambda_{\text {max }}^{\mathrm{EtOH}}$ nm: 242 ( $\varepsilon$ 6700); FAB-MS: $m / z 455[\mathrm{M}+\mathrm{Na}]^{+}, 471[\mathrm{M}+\mathrm{K}]^{+}$; HR-FAB-
MS: Calcd 455.2410 for $\mathrm{C}_{25} \mathrm{H}_{36} \mathrm{O}_{6} \mathrm{Na}$. Found 455.2407.; ${ }^{1} \mathrm{H}$ - and ${ }^{13} \mathrm{C}-\mathrm{NMR}$ : Table 2.

14S $S^{*}, 15$-Epoxyvibsanin C (5) Colorless oil; $[\alpha]_{\mathrm{D}}^{20}+86.0^{\circ}(c=0.12$, $\left.\mathrm{CHCl}_{3}\right)$; IR $v_{\text {max }}^{\mathrm{FT}}: 3491(\mathrm{OH}), 1728(\mathrm{C}=\mathrm{O}), 1649(\mathrm{C}=\mathrm{C}) \mathrm{cm}^{-1}$; UV $\lambda_{\mathrm{max}}^{\mathrm{EtOH}}$ nm: 241 ( $\varepsilon$ 7200); FAB-MS: $m / z 455[\mathrm{M}+\mathrm{Na}]^{+}, 471[\mathrm{M}+\mathrm{K}]^{+}$; HR-FABMS: Calcd 455.2410 for $\mathrm{C}_{25} \mathrm{H}_{36} \mathrm{O}_{6} \mathrm{Na}$. Found 455.2403.; ${ }^{1} \mathrm{H}$ and ${ }^{13} \mathrm{C}-\mathrm{NMR}$ : Table 2 .

Acknowledgements We would like to thank Dr. Masami Tanaka and Miss Yasuko Okamoto (TBU) for NMR and MS measurements, respectively.

\section{References}

1) “The World of Plants," Asahi News Paper Press, Tokyo, 1994, p. 278.

2) Kuroyanagi M., Shiotsu M., Ebihara T., Kawai H., Ueno A., Fukushima S., Chem. Pharm. Bull., 34, 4012-4017 (1986).

3) Kikuchi M., Matsuda N., Sugimoto T., Natural Medicines, 49, 219 (1995).

4) Machida K., Kikuchi M., Tetrahedron Lett., 37, 4157-4160 (1996).

5) Iwagawa T., Yaguchi S., Hase T., Okubo T., Kim M., Phytochemistry, 31, 1311-1315 (1992).

6) Hase T., Iwagawa T., Chem. Lett., 1982, 13-14.

7) Hase T., Takao H., Iwagawa T., Phytochemistry, 22, 1977-1982 (1983).

8) Jensen S. R., Nielsen B. J., Norn V., Phytochemistry, 24, 487-489 (1985).

9) Baker W., Finch A. C. M., Ollis W. D., Robinson K. W., J. Chem. Soc., 1963, 1477-1490.

10) Hase T., Iwagawa T., Bull. Chem. Soc. Jpn., 55, 3663-3664 (1982).

11) Kawazu K., Agric. Biol. Chem., 44, 1367-1372 (1980).

12) Fukuyama Y., Minami H., Takeuchi K., Kodama M., Kawazu K., Tetrahedron Lett., 37, 6767-6776 (1996).

13) Minami H., Anzaki S., Kubo M., Kodama M., Kawazu K., Fukuyama Y., Chem. Pharm. Bull., 46, 1194-1198 (1998).

14) Fukuyama Y., Minami H., Takaoka S., Kodama M., Kawazu K., Nemoto H., Tetrahedron Lett., 38, 1435-1438 (1997).

15) Fukuyama Y., Minami H., Kagawa M., Kodama M., Kawazu K., J. Nat. Prod., 62, 337-339 (1999).

16) Fukuyama Y., Minami H., Yamamoto I., Kodama M., Kawazu K., Chem. Pharm.Bull., 46, 545-547 (1998).

17) Kubo M., Minami H., Hayashi E., Kodama M., Kawazu K., Fukuyama Y., Tetrahedron Lett., 40, 6261-6265 (1999).

18) Kubo M., Chen I. S., Minami H., Fukuyama Y., Chem. Pharm. Bull., 47, 295-296 (1999).

19) Hatsushima S., "Flora of the Ryukyus," Okinawa Biological Education, Naha, 1975 , p. 589 